\title{
CONIVEAU AND THE GROTHENDIECK GROUP OF VARIETIES
}

\author{
DONU ARAPURA AND SU-JEONG KANG
}

There are two natural filtrations on the singular cohomology of a complex smooth projective variety: the coniveau filtration which is defined geometrically, and the level filtration which is defined Hodge theoretically. We will say that the generalized Hodge conjecture (GHC) holds for a variety $X$ if these filtrations coincide on its cohomology. There are a number of intermediate forms of this condition, including the statement that the ordinary Hodge conjecture holds for $X$. We show that if GHC (or an intermediate version of it) holds for $X$ then it holds for any variety $Y$ which defines the same class in a completion of Grothendieck group of varieties. In particular, using motivic integration we see that this is the case if $X$ and $Y$ are birationally equivalent Calabi-Yau varieties, or more generally $K$-equivalent varieties. This refines a result obtained in [A] by a different method.

The key point is to show that the singular cohomology with its coniveau (respectively level) filtration determines a homomorphism $\nu$ (respectively $\lambda$ ) from the Grothendieck group of varieties $\mathrm{K}_{0}\left(\mathcal{V} r_{\mathbb{C}}\right)$ to the Grothendieck group of polarizable filtered Hodge structures $\mathrm{K}_{0}(\mathcal{F H} \mathcal{H})$. This is done by showing that cohomology together with these filtrations behave appropriately under blow ups. We then show that $X$ satisfies $\mathrm{GHC}$ if and only if its class $[X]$ lies in the kernel of the difference $\nu-\lambda$, and the above results follow from this.

The following conventions will be used throughout the paper. All our varieties will be defined over $\mathbb{C}$. We denote the singular cohomology of a smooth projective variety $X$ with rational coefficients by $H^{i}(X)$. Our thanks to the referee for a number of helpful suggestions.

\section{Filtered Hodge structures}

Let $X$ be a smooth projective variety. Its cohomology carries a natural Hodge structure. The coniveau filtration on $H^{i}(X)$ is given by

$$
N^{p} H^{i}(X)=\sum_{\text {codim } S \geq p} \operatorname{ker}\left[H^{i}(X) \rightarrow H^{i}(X-S)\right]
$$

It is a descending filtration by sub Hodge structures. The largest rational sub Hodge structure $\mathcal{F}^{p} H^{i}(X)$ contained in $F^{p} H^{i}(X)$ gives a second filtration, which we call the level filtration. We have $N^{p} H^{i}(X) \subseteq \mathcal{F}^{p} H^{i}(X)$. We will say that $\operatorname{GHC}\left(H^{i}(X), p\right)$ holds if $N^{p} H^{i}(X)=\mathcal{F}^{p} H^{i}(X)$. We will say that generalized Hodge conjecture (GHC) holds for $X$ if we have equality for all $i$ and $p$. Note that $\operatorname{GHC}\left(H^{2 p}(X), p\right)$ is just the usual Hodge conjecture.

We recall that a Hodge structure is polarizable if it admits a polarization, that is a bilinear form satisfying the Hodge-Riemann bilinear relations. We note that the Hodge structure on the cohomology of a smooth projective variety is polarizable:

First author partially supported by the NSF. 
once an ample line bundle is chosen, a polarization is given by taking the orthogonal direct sum of the polarizations, determined in the usual way, on primitive cohomology W] p. 202, 207]. Let $\mathcal{H} \mathcal{S}$ be the category of finite direct sums of pure rational polarizable Hodge structures. The category $\mathcal{H} \mathcal{S}$ is a semisimple Abelian category with tensor products $\mathrm{D}, 4.2 .3]$. Any object $H$ can be decomposed into a sum

$$
H=\bigoplus_{i} H^{i}
$$

where $H^{i}$ is the largest sub Hodge structure of weight $i$. We define the category $\mathcal{F} \mathcal{H} \mathcal{S}$ whose objects are polarizable Hodge structures with finite descending filtrations by sub Hodge structures, and whose morphisms preserve the filtration. Note that this would be a filtration by polarizable sub Hodge structures, since a sub Hodge structure of a polarizable Hodge structure is again polarizable.

Given an additive category $\mathcal{C}$, we can define a Grothendieck group $K_{0}^{\text {split }}(\mathcal{C})$ by generators and relations as follows. We have one generator $[X]$ for each isomorphism class of objects $X \in \mathcal{C}$, and we impose the relation $\left[X_{3}\right]=\left[X_{1}\right]+\left[X_{2}\right]$, whenever $X_{3} \cong X_{1} \oplus X_{2}$. When the category $\mathcal{C}$ possesses exact sequences, we can define a quotient $\mathrm{K}_{0}(\mathcal{C})$ by imposing the above relation when $X_{3}$ is an extension of $X_{2}$ by $X_{1}$. Although it is not strictly necessary for our purposes, we will show in the appendix that these constructions lead to the same groups when applied to $\mathcal{H} \mathcal{S}$ and $\mathcal{F H} \mathcal{H}$. Consequently, we will usually drop the label "split" in the sequel.

By definition, any additive invariant on $\mathcal{H} \mathcal{S}$ or $\mathcal{F H} \mathcal{H}$ factors through their Grothendieck groups. In particular, this remark applies to the Poincaré polynomial

$$
P_{H}(t)=\sum_{i} \operatorname{dim} H^{i} t^{i} \in \mathbb{Z}\left[t, t^{-1}\right]
$$

and a filtered version of it

$$
F P_{(H, N)}(t, u)=\sum_{i, p} \operatorname{dim}\left(N^{p} \cap H^{i}\right) t^{i} u^{p} \in \mathbb{Z}\left[t^{ \pm 1}, u^{ \pm 1}\right]
$$

We can define two functors between the categories $\mathcal{H} \mathcal{S}$ and $\mathcal{F} \mathcal{H} \mathcal{S}$ :

$$
\begin{aligned}
& \Gamma \quad: \quad \mathcal{H} \mathcal{S} \longrightarrow \mathcal{F H} \mathcal{H} \mathcal{S} ; H \longmapsto\left(H, \mathcal{F}^{\bullet}\right) \\
& \Phi \quad: \quad \mathcal{F H} \longrightarrow \mathcal{H} \mathcal{H} \text { S }\left(H, N^{\bullet}\right) \longmapsto H
\end{aligned}
$$

where $\mathcal{F}^{\bullet}$ is the level filtration on $H$, i.e. $\mathcal{F}^{p} H$ is the largest sub Hodge structure of $F^{p} H$. These functors are clearly additive. Thus we obtain well-defined group homomorphisms $\gamma$ and $\phi$, respectively:

$$
\begin{array}{lll}
\gamma & : & \mathrm{K}_{0}(\mathcal{H S}) \longrightarrow \mathrm{K}_{0}(\mathcal{F H} \mathcal{H}) ;[H] \longmapsto\left[\left(H, \mathcal{F}^{\bullet}\right)\right] \\
\phi & : & \mathrm{K}_{0}(\mathcal{F H} \mathcal{H}) \longrightarrow \mathrm{K}_{0}(\mathcal{H S}) ;\left[\left(H, N^{\bullet}\right)\right] \longmapsto[H]
\end{array}
$$

Let $K_{0}\left(\right.$ Var $\left._{\mathbb{C}}\right)$ denote the Grothendieck group of the category of varieties over $\mathbb{C}$ DL1. A more convenient description for our purposes is provided by $\mathrm{B}$, Theorem $3.1]$ :

$$
\mathrm{K}_{0}\left(\operatorname{Var}_{\mathbb{C}}\right) \cong \mathrm{K}_{0}^{\mathrm{bl}}\left(\operatorname{Var}_{\mathbb{C}}\right)
$$

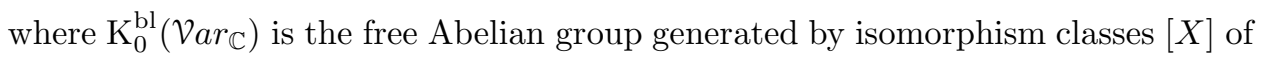
smooth projective varieties subject to the relation $\left[\mathrm{Bl}_{Z} X\right]-[E]=[X]-[Z]$ for every blow up $\mathrm{Bl}_{Z} X$ of $X$ along a smooth closed subvariety $Z \subset X$ with the exceptional divisor $E$. 
Let $X$ be a smooth projective variety. Set

$$
\begin{aligned}
{[X]_{\mathcal{H S}} } & :=\sum_{i}(-1)^{i}\left[H^{i}(X)\right] \in \mathrm{K}_{0}(\mathcal{H S}) \\
{[X]_{\mathcal{F H} \mathcal{S}} } & :=\sum_{i}(-1)^{i}\left[\left(H^{i}(X), N^{\bullet}\right)\right] \in \mathrm{K}_{0}(\mathcal{F H} \mathcal{H})
\end{aligned}
$$

where $N^{\bullet}$ is the coniveau filtration. In the next section, we will show that these classes depend only on $[X] \in \mathrm{K}_{0}^{\mathrm{bl}}\left(\operatorname{Var}_{\mathbb{C}}\right)$.

\section{Coniveau of a Blow up}

We use the following notation throughout this section. Let $X$ be a smooth projective variety and let $\sigma: \tilde{X}=\mathrm{Bl}_{Z} X \rightarrow X$ be the blow up of $X$ along a smooth closed subvariety $Z$ of $X$ of codimension $\geq 2$. The exceptional divisor $E$ can be identified with $\mathbb{P}\left(N_{Z / X}\right)$, where $N_{Z / X}$ is the normal bundle. Therefore it has a tautological line bundle $\mathcal{O}_{E}(1)$. Let $r=\operatorname{codim}(Z, X)-1=\operatorname{dim} E-\operatorname{dim} Z$ and let $h=c_{1}\left(\mathcal{O}_{E}(1)\right)$.



where $i, i_{1}, j, j_{1}$ are inclusions.

\section{Lemma 2.1.}

$N^{p} H^{i}(E)=\sigma^{*}\left(N^{p} H^{i}(Z)\right) \oplus\left(h \cup \sigma^{*}\left(N^{p-1} H^{i-2}(Z)\right)\right) \oplus \cdots \oplus\left(h^{r} \cup \sigma^{*}\left(N^{p-r} H^{i-2 r}(Z)\right)\right)$

Proof. Since $h \in N^{1} H^{2}(E), h^{k} \in N^{k} H^{2 k}(E)$ for each $k \geq 1$. Hence for any $\alpha_{k} \in \sigma^{*}\left(N^{p-k} H^{i-2 k}(Z)\right) \subseteq N^{p-k} H^{i-2 k}(E)$, we have

$$
h^{k} \cup \alpha_{k} \in N^{k} H^{2 k}(E) \cup N^{p-k} H^{i-2 k}(E) \subseteq N^{p} H^{i}(E)
$$

by [AK Corollary 1.2] for $1 \leq k \leq r$. Hence this and $\sigma^{*}\left(N^{p} H^{i}(Z)\right) \subseteq N^{p} H^{i}(E)$ give

$N^{p} H^{i}(E) \supseteq \sigma^{*}\left(N^{p} H^{i}(Z)\right) \oplus\left(h \cup \sigma^{*}\left(N^{p-1} H^{i-2}(Z)\right)\right) \oplus \cdots \oplus\left(h^{r} \cup \sigma^{*}\left(N^{p-r} H^{i-2 r}(Z)\right)\right)$

To show the converse, first note that we have [Le, Proposition 8.23]

$$
H^{i}(E)=\sigma^{*}\left(H^{i}(Z)\right) \oplus\left(\bigoplus_{k=1}^{r}\left(h^{k} \cup \sigma^{*}\left(H^{i-2 k}(Z)\right)\right)\right)
$$

Hence for any $\alpha \in N^{p} H^{i}(E)$, we can decompose

$$
\alpha=\sigma^{*}\left(\alpha_{0}\right)+\left(h \cup \sigma^{*}\left(\alpha_{1}\right)\right)+\left(h^{2} \cup \sigma^{*}\left(\alpha_{2}\right)\right)+\cdots+\left(h^{r} \cup \sigma^{*}\left(\alpha_{r}\right)\right)
$$

where $\alpha_{k} \in H^{i-2 k}(Z)$ for each $k=0, \ldots, r$. We will show that $\alpha_{k} \in N^{p-k} H^{i-2 k}(Z)$ for each $k=0, \ldots, r$ by using descending induction on $k$.

First note that

$$
\sigma_{*}\left(h^{i}\right)= \begin{cases}0 & \text { if } i<r \\ 1 & \text { if } i=r\end{cases}
$$

Therefore, by the projection formula and AK, Theorem 1.1.(1)],

$$
\alpha_{r}=\sigma_{*}\left(h^{r} \cup \sigma^{*}\left(\alpha_{r}\right)\right)=\sigma_{*}(\alpha) \in N^{p-r} H^{i-2 r}(Z)
$$


This shows the claim when $k=r$. Now suppose that $\alpha_{l} \in N^{p-l} H^{i-2 l}(Z)$ for $k+1 \leq l \leq r$. We have to show that $\alpha_{k} \in N^{p-k} H^{i-2 k}(Z)$. By assumption, for $k+1 \leq l \leq r$ we have

$$
h^{l} \cup \sigma^{*}\left(\alpha_{l}\right) \in N^{l} H^{2 l}(E) \cup N^{p-l} H^{i-2 l}(E) \subseteq N^{p} H^{i}(E)
$$

Set

$$
\beta_{k+1}=\alpha-\sum_{l=k+1}^{r}\left(h^{l} \cup \sigma^{*}\left(\alpha_{l}\right)\right)
$$

Then, we have

$$
\beta_{k+1}=\sigma^{*}\left(\alpha_{0}\right)+\left(h \cup \sigma^{*}\left(\alpha_{1}\right)\right)+\cdots+\left(h^{k} \cup \sigma^{*}\left(\alpha_{k}\right)\right) \in N^{p} H^{i}(E)
$$

Then by taking a cup product with $h^{r-k} \in N^{r-k} H^{2(r-k)}(E)$, we get

$$
\begin{aligned}
h^{r-k} \cup \beta_{k+1} & =\left(h^{r-k} \cup \sigma^{*}\left(\alpha_{0}\right)\right)+\left(h^{r-k+1} \cup \sigma^{*}\left(\alpha_{1}\right)\right)+\cdots+\left(h^{r-k+k} \cup \sigma^{*}\left(\alpha_{k}\right)\right) \\
& \in N^{p+(r-k)} H^{i+2(r-k)}(E)
\end{aligned}
$$

Then,

$$
\sigma_{*}\left(h^{r-k} \cup \beta_{k+1}\right)=\sigma_{*}\left(h^{r} \cup \sigma^{*}\left(\alpha_{k}\right)\right)=\alpha_{k} \in N^{p-k} H^{i-2 k}(Z)
$$

as we claimed. Therefore we have

$N^{p} H^{i}(E) \subseteq \sigma^{*}\left(N^{p} H^{i}(Z)\right) \oplus\left(h \cup \sigma^{*}\left(N^{p-1} H^{i-2}(Z)\right)\right) \oplus \cdots \oplus\left(h^{r} \cup \sigma^{*}\left(N^{p-r} H^{i-2 r}(Z)\right)\right)$

Corollary 2.2. Given $\alpha \in N^{p} H^{i}(E)$, we can write

$$
\alpha=\sigma^{*}\left(\alpha_{0}\right)+(h \cup \beta)
$$

where $\alpha_{0} \in N^{p} H^{i}(Z)$ and $\beta \in N^{p-1} H^{i-2}(E)$

The following is well known, but we indicate the proof for lack of a suitable reference.

Lemma 2.3. There is a short exact sequence of pure Hodge structures of weight $i$ :

$$
0 \longrightarrow H^{i}(X) \stackrel{\sigma^{*}+i^{*}}{\longrightarrow} H^{i}(\tilde{X}) \oplus H^{i}(Z) \stackrel{-j^{*}+\sigma^{*}}{\longrightarrow} H^{i}(E) \longrightarrow 0
$$

Proof. We have a commutative diagram with exact rows:

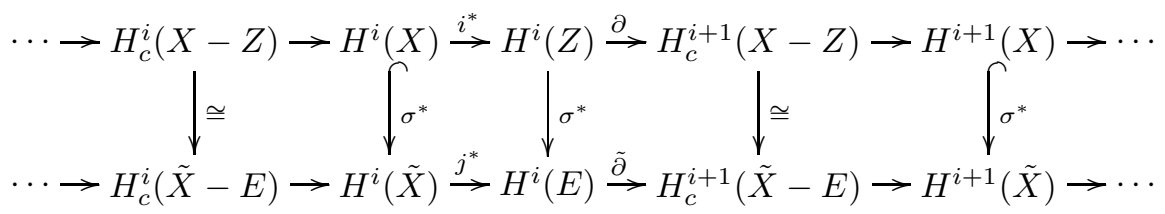

where some of the arrows are injections or isomorphisms as indicated. The lemma now follows from a straight forward diagram chase.

Lemma 2.4. The sequence of the previous lemma is exact in $\mathcal{F H} \mathcal{H}$, i.e.

$$
0 \longrightarrow N^{p} H^{i}(X) \longrightarrow N^{p} H^{i}(\tilde{X}) \oplus N^{p} H^{i}(Z) \longrightarrow N^{p} H^{i}(E) \longrightarrow 0
$$

is an exact sequence for all $p$. 
Proof. Consider the short exact sequence (11) and note that

$$
\begin{gathered}
\left(\sigma^{*}+i^{*}\right)\left(N^{p} H^{i}(X)\right) \subseteq N^{p} H^{i}(\tilde{X}) \oplus N^{p} H^{i}(Z), \\
\left(-j^{*}+\sigma^{*}\right)\left(N^{p} H^{i}(\tilde{X}) \oplus N^{p} H^{i}(Z)\right) \subseteq N^{p} H^{i}(E)
\end{gathered}
$$

since all maps preserve the coniveau.

We will check exactness in the middle. It suffices to show that

$$
\left.\left.\operatorname{im}\left(\sigma^{*}+i^{*}\right)\right|_{N^{p} H^{i}(X)} \supseteq \operatorname{ker}\left(-j^{*}+\sigma^{*}\right)\right|_{N^{p} H^{i}(\tilde{X}) \oplus N^{p} H^{i}(Z)},
$$

since the reverse inclusion follows from (11). Let $(\beta, \gamma) \in N^{p} H^{i}(\tilde{X}) \oplus N^{p} H^{i}(Z)$ such that $(\beta, \gamma) \in \operatorname{ker}\left(-j^{*}+\sigma^{*}\right)$. Then, from the exact sequence (1), there is $\alpha \in H^{i}(X)$ such that $\left(\sigma^{*}+i^{*}\right)(\alpha)=(\beta, \gamma)$. In particular, we have

$$
\sigma^{*}(\alpha)=\beta \in N^{p} H^{i}(\tilde{X})
$$

Since $\sigma: \tilde{X} \rightarrow X$ is a birational map, we have

$$
\alpha=\left(\sigma_{*} \circ \sigma^{*}\right)(\alpha)=\sigma_{*}(\beta) \in \sigma_{*}\left(N^{p} H^{i}(\tilde{X})\right) \subseteq N^{p} H^{i}(X)
$$

Hence we have $(\beta, \gamma)=\left(\sigma^{*}+i^{*}\right)(\alpha) \in\left(\sigma^{*}+i^{*}\right)\left(N^{p} H^{i}(X)\right)$.

We will check surjectivity of $\left.\left(-j^{*}+\sigma^{*}\right)\right|_{N^{p} H^{i}(\tilde{X}) \oplus N^{p} H^{i}(Z)}$. Let $\alpha \in N^{p} H^{i}(E)$. Then by Corollary 2.2 we can decompose

$$
\alpha=\sigma^{*}\left(\alpha_{0}\right)+(h \cup \beta)
$$

where $\alpha_{0} \in N^{p} H^{i}(Z)$ and $\beta \in N^{p-1} H^{i-2}(E)$. Note the composition

$$
H^{i-2}(E) \stackrel{j_{*}}{\longrightarrow} H^{i}(\tilde{X}) \stackrel{j^{*}}{\longrightarrow} H^{i}(E)
$$

is same as cupping with $\left.[E]\right|_{E}$. Since $\left.\mathcal{O}(E)\right|_{E}=\mathcal{O}_{E}(-1)$ and $h=c_{1}\left(\mathcal{O}_{E}(1)\right)$, we have

$$
\left(j^{*} \circ j_{*}\right)(\beta)=\left.[E]\right|_{E} \cup \beta=-h \cup \beta
$$

Thus, we have

$$
\alpha=\sigma^{*}\left(\alpha_{0}\right)-j^{*}\left(j_{*}(\beta)\right)
$$

and $j_{*}(\beta) \in j_{*}\left(N^{p-1} H^{i-2}(E)\right) \subseteq N^{p} H^{i}(\tilde{X})$ and this shows that the map $\left(-j^{*}+\right.$ $\left.\sigma^{*}\right)\left.\right|_{N^{p} H^{i}(\tilde{X}) \oplus N^{p} H^{i}(Z)}$ is surjective.

Let $f: X \rightarrow Y$ be a morphism of smooth projective varieties. Then $f^{*}$ preserves the coniveau and induces maps $\bar{f}^{*}: G r_{N}^{p} H^{i}(Y) \rightarrow G r_{N}^{p} H^{i}(X)$.

Corollary 2.5. The following sequence is exact:

$$
0 \longrightarrow G r_{N}^{p} H^{i}(X) \longrightarrow G r_{N}^{p} H^{i}(\tilde{X}) \oplus G r_{N}^{p} H^{i}(Z) \longrightarrow G r_{N}^{p} H^{i}(E) \longrightarrow 0
$$

Proof. This follows from lemma A.1

We want to construct well-defined morphisms from $\mathrm{K}_{0}^{\mathrm{bl}}\left(\operatorname{Var}_{\mathbb{C}}\right)$ to $\mathrm{K}_{0}(\mathcal{H} \mathcal{S})$ and from $\mathrm{K}_{0}^{\mathrm{bl}}\left(\mathcal{V}_{\mathrm{C}} r_{\mathbb{C}}\right)$ to $\mathrm{K}_{0}(\mathcal{F} \mathcal{H} \mathcal{S})$. First we need the following lemmas.

Lemma 2.6. The relation

$$
\left[B l_{Z} X\right]_{\mathcal{H S}}-[E]_{\mathcal{H S}}=[X]_{\mathcal{H S}}-[Z]_{\mathcal{H S}}
$$

holds. Hence, we have a well-defined group homomorphism

$$
K_{0}^{b l}\left(\mathcal{V a r}_{\mathbb{C}}\right) \longrightarrow K_{0}(\mathcal{H S}) ;[X] \mapsto[X]_{\mathcal{H S}}
$$


Proof. By Lemma 2.3 we have a short exact sequence of pure Hodge structures:

$$
0 \longrightarrow H^{i}(X) \longrightarrow H^{i}(\tilde{X}) \oplus H^{i}(Z) \longrightarrow H^{i}(E) \longrightarrow 0
$$

Then by the definition of $\mathrm{K}_{0}(\mathcal{H} \mathcal{S})$, we have

$$
\left[H^{i}(X)\right]+\left[H^{i}(E)\right]=\left[H^{i}(\tilde{X}) \oplus H^{i}(Z)\right]=\left[H^{i}(\tilde{X})\right]+\left[H^{i}(Z)\right]
$$

and the result follows immediately by taking alternating sums.

We define

$$
\lambda: \mathrm{K}_{0}^{\mathrm{bl}}\left(\mathcal{V a r}_{\mathbb{C}}\right) \longrightarrow \mathrm{K}_{0}(\mathcal{F H} \mathcal{H})
$$

as the composition of the map constructed in the above lemma with $\gamma$.

Lemma 2.7. The relation

$$
\left[B l_{Z} X\right]_{\mathcal{F H S}}-[E]_{\mathcal{F H} \mathcal{S}}=[X]_{\mathcal{F H} \mathcal{H}}-[Z]_{\mathcal{F H} \mathcal{S}}
$$

holds. Hence we have a well-defined group homomorphism

$$
\nu: K_{0}^{b l}\left(\mathcal{V a r}_{\mathbb{C}}\right) \longrightarrow K_{0}(\mathcal{F H S}) ; \nu([X])=[X]_{\mathcal{F H}}
$$

Proof. We showed in Lemma 2.4 that

$$
0 \rightarrow\left[\left(H^{i}(X), N^{\bullet}\right)\right] \rightarrow\left[\left(H^{i}(\tilde{X}) \oplus H^{i}(Z), N^{\bullet}\right)\right] \rightarrow\left[\left(H^{i}(E), N^{\bullet}\right)\right] \rightarrow 0
$$

is exact in $\mathcal{F H} \mathcal{H}$ shere $N^{p}\left(H^{i}(\tilde{X}) \oplus H^{i}(Z)\right)=N^{p} H^{i}(\tilde{X}) \oplus N^{p} H^{i}(Z)$. The rest of the argument is exactly the same as above.

Remark 2.8. The proof of Lemma 2.4 shows that the above sequence is split exact. So in particular, as the referee has pointed to us, we can construct the homomorphism

$$
\nu: K_{0}^{b l}\left(\mathcal{V a r}_{\mathbb{C}}\right) \longrightarrow K_{0}^{\text {split }}(\mathcal{F} \mathcal{H S})
$$

directly, without appealing to the results of the appendix.

\section{MAIN THEOREM}

Theorem 3.1. Let $X$ be a smooth projective variety. Then, the following statements are equivalent:

1. GHC holds for $X$.

2. $[X] \in \operatorname{ker}(\nu-\lambda)$.

3. The equality $F P_{[X]_{\mathcal{F H}}}(t, u)=F P_{\gamma\left([X]_{\mathcal{H} S}\right)}(t, u)$ of filtered Poincaré polynomials holds.

Proof. It is clear that GHC for $X$ implies $[X]_{\mathcal{F H S}}=\gamma\left([X]_{\mathcal{H S}}\right)$, or equivalently that $[X] \in \operatorname{ker}(\nu-\lambda)$.

Suppose $[X] \in \operatorname{ker}(\nu-\lambda)$. Then

$$
[X]_{\mathcal{F H S}}=\gamma\left([X]_{\mathcal{H S}}\right)
$$

i.e.

$$
\sum_{i}(-1)^{i}\left[\left(H^{i}(X), N^{\bullet}\right)\right]=\sum_{i}(-1)^{i}\left[\left(H^{i}(X), \mathcal{F}^{\bullet}\right)\right]
$$

Taking the filtered Poincaré polynomial $F P(t, u)$ of both sides yields the third statement. 
Assume the equality in 3 . The coefficient of $t^{i}$ on the left is

$$
(-1)^{i} \sum_{p} \operatorname{dim} N^{p} H^{i}(X) u^{p}
$$

while on the right it is

$$
(-1)^{i} \sum_{p} \operatorname{dim} \mathcal{F}^{p} H^{i}(X) u^{p}
$$

The equality of these expressions forces $N^{p} H^{i}(X)=\mathcal{F}^{p} H^{i}(X)$ in this case.

Remark 3.2. The coefficient of $t^{i} u^{p}$ in $F P_{[X]_{\mathcal{F H}}}(t, u)$ is $(-1)^{i} \operatorname{dim} N^{p} H^{i}(X)$, and the coefficient of $t^{i} u^{p}$ in $F P_{\gamma\left([X]_{\mathcal{H}}\right)}(t, u)$ is $(-1)^{i} \operatorname{dim} \mathcal{F}^{p} H^{i}(X)$. Therefore $G H C\left(H^{i}(X), p\right)$ holds precisely when these coefficients coincide.

Let $\mathbb{L}^{\prime}=\left[\mathbb{A}_{\mathbb{C}}^{1}\right] \in \mathrm{K}_{0}\left(\operatorname{Var}_{\mathbb{C}}\right)$ denote the Lefschetz object. Under the isomorphism

$$
\mathrm{K}_{0}\left(\operatorname{Var}_{\mathbb{C}}\right) \cong \mathrm{K}_{0}^{\mathrm{bl}}\left(\operatorname{Var}_{\mathbb{C}}\right)
$$

$\mathbb{L}^{\prime}$ maps to $\mathbb{L}=\left[\mathbb{P}^{1}\right]-[\infty]$. Let

$$
\mathbb{K}=\left[\left(\mathbb{Q}(-1), N^{\bullet}\right)\right]
$$

where $N^{\bullet}$ is the filtration determined by $N^{1} / N^{2}=\mathbb{Q}(-1)$. Then

$$
\lambda(\mathbb{L})=\nu(\mathbb{L})=\mathbb{K}
$$

We have a product on the category $\mathcal{F H} \mathcal{H}$ which is just the tensor product filtered by

$$
N^{p}\left(H_{1} \otimes H_{2}\right)=\bigoplus_{r+s=p} N^{r} H_{1} \otimes N^{s} H_{2}
$$

This gives a commutative ring structure on $\mathrm{K}_{0}(\mathcal{F H} \mathcal{H} \mathcal{S})$. The group $\mathrm{K}_{0}^{\mathrm{bl}}\left(\operatorname{Var}_{\mathbb{C}}\right)$ also has a commutative ring structure induced by the product of varieties. It is not clear whether $\lambda$ or $\nu$ are ring homomorphisms, however we do have:

Lemma 3.3. For any $\eta \in K_{0}^{b l}\left(\operatorname{Var}_{\mathbb{C}}\right)$,

$$
\begin{aligned}
& \lambda(\mathbb{L} \cdot \eta)=\mathbb{K} \cdot \lambda(\eta) \\
& \nu(\mathbb{L} \cdot \eta)=\mathbb{K} \cdot \nu(\eta)
\end{aligned}
$$

Proof. Under the Künneth isomorphism, we have

$$
N^{p} H^{i}\left(\mathbb{P}^{1} \times X\right) \cong N^{p-1} H^{i-2}(X)(-1) \oplus N^{p} H^{i}(\{\infty\} \times X),
$$

and a similar statement holds for $\mathcal{F}^{p}$. The lemma is an immediate consequence.

The element $\mathbb{K}$ is invertible, and the above identities guarantee that $\lambda$ or $\nu$ factor through the localization $\mathcal{M}=\mathrm{K}_{0}^{\mathrm{bl}}\left(\operatorname{Var}_{\mathbb{C}}\right)\left[\mathbb{L}^{-1}\right]$.

Recall (DL1) that there is a decreasing filtration $F^{\bullet} \mathcal{M}$ on $\mathcal{M}$, where $F^{m} \mathcal{M}$ is the subgroup of $\mathcal{M}$ generated by $\left\{[X] \cdot \mathbb{L}^{-j} \mid \operatorname{dim} X-j \leq-m\right\}$. Let $\widehat{\mathcal{M}}$ be the completion of the ring $\mathcal{M}$ with respect to the filtration $F^{\bullet} \mathcal{M}$. A similar filtration (compare [Loo ) can be defined on $\mathrm{K}_{0}(\mathcal{F H} \mathcal{H})$ by replacing dimension by weights. More precisely, let $L^{m} \mathrm{~K}_{0}(\mathcal{F H} \mathcal{H})$ be the subgroup generated by $\left\{\left[\left(H, N^{\bullet}\right)\right] \mid \max \{i \mid\right.$ $\left.\left.H^{i} \neq 0\right\} \leq-m\right\}$. We denote the completion of $\mathrm{K}_{0}(\mathcal{F H} \mathcal{H} \mathcal{S})$ with respect to $L^{\bullet}$ by $\widehat{\mathcal{N}}$. The weights of the Hodge structure on cohomology of a smooth projective variety of dimension $\leq d$ are bounded by $2 d$. Therefore the induced filtrations $\lambda\left(F^{\bullet}\right)$ and 
$\nu\left(F^{\bullet}\right)$ are cofinal with a subfiltration of $L^{\bullet}$. It follows that we have a commutative diagram

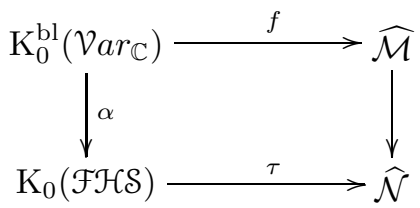

where $\alpha$ denotes either $\lambda$ or $\nu$, and $f, \tau$ are the canonical ones.

Lemma 3.4. The homomorphism $K_{0}(\mathcal{F H} \mathcal{H}) \rightarrow \mathbb{Z}\left[t^{ \pm 1}, u^{ \pm 1}\right]$ given by the filtered Poincaré polynomial factors through the image of $\tau$.

Proof. Let $\eta \in \bigcap_{m} L^{m}$. Then for each $m \in \mathbb{Z}, \eta$ can be expressed as a linear combination of classes of filtered Hodge structures of weight at most $-m$. Thus the degree of $F P_{\eta}(t, u)$ in $t$ is bounded above by $-m$, for all $m \in \mathbb{Z}$. This is impossible unless $F P_{\eta}(t, u)=0$.

Corollary 3.5 (to theorem). Let $X, Y$ be smooth projective varieties such that $X, Y$ define the same classes in $\widehat{\mathcal{M}}$. Then $G H C$ holds for $X$ if and only if $G H C$ holds for $Y$.

Proof. If the images of $[X]$ and $[Y]$ coincide in $\widehat{\mathcal{M}}$ then they coincide in $\widehat{\mathcal{N}}$. Therefore their filtered Poincaré polynomials coincide. The conclusion is now an immediate consequence of Theorem 3.1

From Remark 3.2 we obtain:

Corollary 3.6. Let $X, Y$ be smooth projective varieties such that $X, Y$ define the same classes in $\widehat{\mathcal{M}}$. Then for each $i$ and $p, \operatorname{GHC}\left(H^{i}(X), p\right)$ holds if and only if $\operatorname{GHC}\left(H^{i}(Y), p\right)$ holds.

The proof of the next corollary depends on the motivic integration theory of Kontsevich, Denef and Loeser. See [DL1], [DL2], and [Loo for an introduction to these ideas.

Corollary 3.7. Let $X, Y$ be $K$-equivalent smooth projective varieties, i.e. there is a smooth projective variety $Z$ and birational maps $\pi_{1}: Z \rightarrow X$ and $\pi_{2}: Z \rightarrow Y$ such that $\pi_{1}^{*} K_{X}=\pi_{2}^{*} K_{Y}$, where $K_{X}$ (respectively $K_{Y}$ ) is the canonical divisor on $X$ (respectively $Y)$. Then $\operatorname{GHC}\left(H^{i}(X), p\right)$ holds if and only if $\operatorname{GHC}\left(H^{i}(Y), p\right)$ holds.

Proof. It is enough to show that $X$ and $Y$ define the same class in $\widehat{\mathcal{M}}$. This follows from the $K$-equivalence assumption by a standard application of motivic integration theory, see [Loe or $\underline{\underline{V}}$. For convenience of the reader, we reproduce the argument. By the change of variables formula [DL1, Lemma 3.3], we have

$$
\begin{aligned}
f([X])=\int_{\mathcal{L}(X)} d \mu_{X} & =\int_{\mathcal{L}(Z)} \mathbb{L}^{-o r d_{t} \pi_{1}^{*} \omega_{X}} d \mu_{Z} \\
& =\int_{\mathcal{L}(Z)} \mathbb{L}^{-o r d_{t} \pi_{2}^{*} \omega_{Y}} d \mu_{Z}=\int_{\mathcal{L}(Y)} d \mu_{Y}=f([Y])
\end{aligned}
$$

where $f: \mathrm{K}_{0}^{\mathrm{bl}}\left(\mathcal{V}_{r_{\mathbb{C}}}\right) \rightarrow \widehat{\mathcal{M}}$ is the canonical map, $\mathcal{L}(X), \mathcal{L}(Y)$ are the arc spaces, $\omega_{X}, \omega_{Y}$ are the canonical sheaves, and $d \mu_{X}, d \mu_{Y}$ are the motivic measures. Hence the corollary follows from Corollary 3.5 
Corollary 3.8. Let $X, Y$ be birational Calabi-Yau varieties. Then $G H C\left(H^{i}(X), p\right)$ holds if and only if $\operatorname{GHC}\left(H^{i}(Y), p\right)$ holds.

Proof. Since $K_{X}=K_{Y}=0$, it follows from Corollary 3.7

\section{Appendix A. GrothendieCK Groups of Filtered CATEGories}

We recall that an exact category consists of an additive category $\mathcal{C}$, together with a distinguished class of diagrams

$$
0 \rightarrow A \rightarrow B \rightarrow C \rightarrow 0
$$

called exact sequences satisfying appropriate conditions Q . For example, any additive category $\mathcal{C}$ can be made exact by taking the class of exact sequences to be isomorphic to the class of split sequences

$$
0 \rightarrow A \rightarrow A \oplus C \rightarrow C \rightarrow 0
$$

An Abelian category gives another example of an exact category, where exact sequences have the usual meaning. If the category is also semisimple, then this exact structure coincides with the split structure above. This remark applies to $\mathcal{H} \mathcal{S}$.

Let $\mathcal{C}$ be an Abelian category, and let $\mathcal{F C}$ (respectively $\mathcal{G} \mathcal{C}$ ) denote the category of filtered (respectively graded) objects in $\mathcal{C}$. The category $\mathcal{G C}$ is Abelian, but $\mathcal{F} \mathfrak{C}$ is generally not. However $\mathcal{F C}$ has a natural exact structure [BBD, 1.1.4] given as follows. We have a functor

$$
\left(H, N^{\bullet}\right) \longmapsto \bigoplus_{p} N^{p} H
$$

from $\mathcal{F C}$ to $\mathcal{G}$. We declare a sequence

$$
0 \rightarrow\left(H_{1}, N^{\bullet}\right) \rightarrow\left(H_{2}, N^{\bullet}\right) \rightarrow\left(H_{3}, N^{\bullet}\right) \rightarrow 0
$$

in $\mathcal{F C}$ to be exact if and only if its image in $\mathcal{G C}$ is exact. For the record, we note the following alternative formulation, which is perhaps more common:

Lemma A.1. The sequence

$$
0 \rightarrow\left(H_{1}, N^{\bullet}\right) \rightarrow\left(H_{2}, N^{\bullet}\right) \rightarrow\left(H_{3}, N^{\bullet}\right) \rightarrow 0
$$

is exact if and only if the following sequence is exact in $\mathrm{GC}$ :

$$
0 \rightarrow \bigoplus_{p} N^{p} H_{1} / N^{p+1} H_{1} \longrightarrow \bigoplus_{p} N^{p} H_{2} / N^{p+1} H_{2} \longrightarrow \bigoplus_{p} N^{p} H_{3} / N^{p+1} H_{3} \rightarrow 0
$$

Proof. This is a straight forward application of the Snake lemma and induction.

Lemma A.2. The category $\mathcal{F} \mathcal{C}$ with the above notion of exact sequence is an exact category.

Given an exact category $\mathcal{C}$, its Grothendieck group $\mathrm{K}_{0}(\mathcal{C})$ is given by generators $[M]$, with $M \in \mathcal{C}$, and relations $\left[M_{2}\right]=\left[M_{1}\right]+\left[M_{3}\right]$ for every exact sequence $0 \rightarrow M_{1} \rightarrow M_{2} \rightarrow M_{3} \rightarrow 0$. Let us denote the Grothendieck group for $\mathcal{C}$ with its split exact structure by $\mathrm{K}_{0}^{\text {split }}(\mathcal{C})$. We see immediately that, $\mathrm{K}_{0}^{\text {split }}(\mathcal{C}) \cong \mathrm{K}_{0}(\mathcal{C})$ if $\mathcal{C}$ is Abelian and semisimple. This is, in particular, the case for $\mathcal{H} \mathcal{S}$. We have a homomorphism $\mathrm{K}_{0}^{\text {split }}(\mathcal{F} \mathcal{H} \mathcal{S}) \rightarrow \mathrm{K}_{0}(\mathcal{F H} \mathcal{H})$, which is also an isomorphism by:

Lemma A.3. If $\mathcal{C}$ is semisimple Abelian, then any exact sequence in $\mathcal{F} \mathcal{C}$ is split exact. 
Proof. It is enough to check that given an exact sequence in $\mathcal{F C}$

$$
0 \rightarrow\left(H_{1}, N^{\bullet}\right) \rightarrow\left(H_{2}, N^{\bullet}\right) \stackrel{f}{\rightarrow}\left(H_{3}, N^{\bullet}\right) \rightarrow 0,
$$

there is a splitting $s:\left(H_{3}, N^{\bullet}\right) \rightarrow\left(H_{2}, N^{\bullet}\right)$ for $f$. First note that by semisimplicity of $\mathcal{C}$, we have a noncanonical decomposition

$$
N^{p} H_{i} \cong N^{p+1} H_{i} \oplus G r_{N}^{p} H_{i}
$$

where $G r_{N}^{p} H_{i}=N^{p} H_{i} / N^{p+1} H_{i}, i=2,3$. We define $s_{p}=\left.s\right|_{N^{p} H_{3}}$ by descending induction on $p$ : Note that the exact sequence (2) induces exact sequences in $\mathcal{C}$

$$
\begin{gathered}
0 \longrightarrow N^{p+1} H_{1} \longrightarrow N^{p+1} H_{2} \stackrel{f_{p+1}}{\longrightarrow} N^{p+1} H_{3} \longrightarrow 0 \\
0 \longrightarrow G r_{N}^{p} H_{1} \longrightarrow G r_{N}^{p} H_{2} \stackrel{\bar{f}_{p}}{\longrightarrow} G r_{N}^{p} H_{3} \longrightarrow 0
\end{gathered}
$$

where $f_{p+1}=\left.f\right|_{N^{p+1} H_{2}}$ and $\bar{f}_{p}$ is the induced map. By induction and semisimplicity of $\mathcal{C}$, there are splittings $s_{p+1}:\left(N^{p+1} H_{3}, N^{\bullet} \cap N^{p+1} H_{3}\right) \rightarrow\left(N^{p+1} H_{2} N^{\bullet} \cap N^{p+1} H_{2}\right)$ and $t_{p}: G r_{N}^{p} H_{3} \rightarrow G r_{N}^{p} H_{2}$ for $f_{p+1}$ and $\bar{f}_{p}$, respectively. Set

$$
s_{p}=s_{p+1}+t_{p}: N^{p} H_{3} \longrightarrow N^{p} H_{2}
$$

Then $s_{p}$ gives a well-defined splitting for $f_{p}$ and hence we have a splitting $s=s_{0}$ for $f$. This completes the proof of the Lemma.

Corollary A.4. $K_{0}^{\text {split }}(\mathcal{F H} \mathcal{H}) \cong K_{0}(\mathcal{F H} \mathcal{H})$

\section{REFERENCES}

[A] D. Arapura, Motivation for Hodge cycles, ArXiV preprint (math.AG/0501348)

[AK] D. Arapura, S-J. Kang, Functoriality of the coniveau filtration, Canadian Math. Bull. (to appear)

[dB] S. del Baño, On the Chow motive of some moduli spaces, J. reine angew. Math. 532 (2001), $105-132$

[BBD] A. Beilinson, J. Bernstein, P. Deligne, Faisceux Pervers, Astérisque No. 100 (1982)

[B] F. Bittner, The universal Euler characteristic for varieties of characteristic zero, Compositio Math. 140 (2004), 1011-1032

[D] P. Deligne, Théorie de Hodge II, Publ. IHES No. 40 (1971), 5-57

[DL1] J. Denef, F. Loeser, Germs of arcs on singular algebraic varieties and motivic integration, Invent. Math. 135 (1999), no. 1, 201-232

[DL2] J. Denef, F. Loeser, Geometry on arc spaces of algebraic varieties, Proc. 3rd European Congress of Math. Barcelona, 327-348 (2001), Birkhäuser.

[G] A. Grothendieck, Hodge's general conjecture is false for trivial reasons, Topology 8, (1969), 299-303

[Le] J. Lewis, A Survey of the Hodge conjecture, 2nd ed. CRM Monograph Series, 10. AMS, (1999)

[Loe] F. Loeser, Notes on p-adic and motivic integration, Arizona winter school (2003), http://www.dma.ens.fr/ loeser

[Loo] E. Looijenga, Motivic measures, Séminaire Bourbaki, Vol. 1999/2000, Astérisque No. 276 (2002), 267-297

[Q] D. Quillen, Higher algebraic K-theory I, Lect. Notes in Math. No. 341, Springer-Verlag (1973), 77-139

[V] W. Veys, Arc spaces, motivic integration and stringy invariants, ArXiV preprint (math.AG/0401374)

[W] R. Wells, Differential analysis on complex manifolds, Springer-Verlag (1980)

Department of Mathematics, Purdue University, West lafayette, IN 47907, U.S.A. 